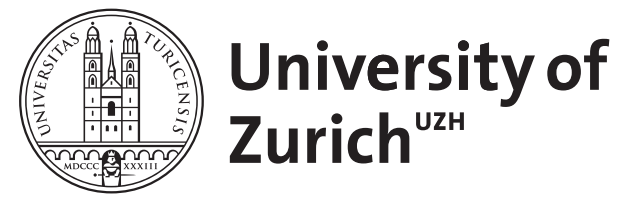

Zurich Open Repository and Archive

University of Zurich

University Library

Strickhofstrasse 39

CH-8057 Zurich

www.zora.uzh.ch

Year: 2018

Die Nachahmung von Sauerstoffmangel als Therapie?

Humar, Rok

DOI: https://doi.org/10.1024/1661-8157/a003082

Posted at the Zurich Open Repository and Archive, University of Zurich

ZORA URL: https://doi.org/10.5167/uzh-158143

Journal Article

Published Version

Originally published at:

Humar, Rok (2018). Die Nachahmung von Sauerstoffmangel als Therapie? Praxis, 107(21):1125.

DOI: https://doi.org/10.1024/1661-8157/a003082 


\section{Die Nachahmung}

\section{von Sauerstoffmangel als Therapie?}

Alle Metazoen, zu denen auch wir uns zählen, brauchen Sauerstoff, um Energie zu erzeugen. Mangelt es an Sauerstoff, können Zellen und Gewebe absterben. Unser Organismus kann jedoch Massnahmen einleiten, die die Gewebe anpassen und schützen.

In dem Mini-Review «Ist Sauerstoffmangel immer nur schädlich?» [1] zeigt M. Schläpfer auf, welchen Schaden Sauerstoffmangel auf zellulärer Ebene anrichtet, wie die Zelle Sauerstoffmangel auf der molekularen Ebene erspürt, und wie sie Gegenmassnahmen einleitet. Diese Massnahmen werden grösstenteils durch Hypoxie-induzierbare Faktoren (HIFs) und Prolylhydroxylasen (PHDs) vermittelt und verbessern die Sauerstoffversorgung nachhaltig, indem die Hämatopoese beschleunigt, neue Blutgefässe gebildet oder die Energieversorgung den widrigen Gegebenheiten angepasst wird. Wir können uns nun, dank Forschung und Entwicklung, diese Gegenmassnahmen zu Nutze machen, um regenerierende Therapien zu entwickeln: Als innovative Therapieoptionen werden in klinischen Studien der Phasen II und III HIF-Stabilisatoren untersucht. Die Hemmung von PHDs ahmt eine Hypoxie nach und führt zu einer erhöhten Expression von HIF-induzierbaren Genprodukten. Die Behandlung von Anämie bei chronischer Nierenkrankheit mit PHD-Inhibitoren steht hier momentan im Mittelpunkt. Nicht nur wird durch PHD-Inhibition die Erythropoetin-Expression gesteigert, auch die Eisenaufnahme durch HIF-1- und HIF-2-gesteuerte Mechanismen wird erhöht, um so synergistisch und gezielt der Anämie entgegen zu wirken.

Mehr als 70 direkte HIF-Zielgene sind bekannt, die sowohl bei physiologischen wie pathologischen Prozessen mitwirken. Wie schon erwähnt, stehen hier Angiogenese und die Versorgung mit Sauerstoff, sowie die Verschiebung des zellulären Energiestoffwechsels von der oxidativen Phosphorylierung zur Glykolyse im Vordergrund.

Aber auch Gene, die Stammzelleigenschaften/Selbsterneuerung, Zellteilung und Apoptose, Redoxhomöostase, epitheliale-mesenchymale Transition, Metastasierung und Invasion steuern, werden durch HIFs aktiviert [2].

Die möglichen Anwendungen von PHD-Inhibitoren bei verschiedenen Erkrankungen sind somit vielfältig und eröffnen eine breite Perspektive, bergen aber auch Gefahren. Laufende klinische Studien sind ermutigend, die Übertragung auf das Patientenbett erfordert aber noch weitere präklinische und klinische Studien, die mehrere Probleme lösen müssen, wie es Harnoss et al. [3] zusammenfassend formulieren:

1. PHD-Inhibitoren sollten mit einer gewissen Selektivität für die einzelnen PHDs sowie für verschiedene Gewebe ausgestattet sein.
2. Der Zeitrahmen für die Anwendung von PHD-Inhibitoren bei der Vorbehandlung und der Behandlung sollten definiert werden, um die Vorteile und möglichen Nebenwirkungen einer konstanten HIF-Stabilisierung auszugleichen.

3. Alternative PHD-Inhibitor-Anwendungswege, wie z. B. lokale Anwendung bei der Wundheilung, sind wünschenswert, um systemische Nebenwirkungen zu minimieren.

4. Schliesslich, wie M. Schläpfer ausführt, stammen die profundesten Erkenntnisse über die HIF/PHD-Achse aus der Tumorbiologie: Obwohl PHD-Inhibitoren, die zur Zeit in Phase III geprüft werden, in vorklinischen Versuchen zu keiner erhöhten Karzinogenität führten [4], ist die Bedeutung von PHD-Inhibitoren bei der Tumorgenese (entweder tumorunterdrückend als Antikrebs-Therapeutika oder tumorfördernd als eine schwere systemische Nebenwirkung) leider immer noch schwer zu erfassen und muss in weiteren klinischen Studien geklärt werden $[3,5]$.

Können diese Fragen aber ausreichend beantwortet werden, so steht uns durch PHD-Inhibitoren eine vielversprechende, neuartige Gruppe von Therapeutika zur Verfügung, die ihre Wirkung über die zelleigenen Schutzmechanismen entfaltet.

\section{Bibliografie:}

1. Schläpfer M: Ist Sauerstoffmangel immer nur schädlich? Praxis 2018; 107: 1155-1159.

2. Dengler VL, Galbraith M, Espinosa JM: Transcriptional regulation by hypoxia inducible factors. Crit Rev Biochem Mol Biol 2014; 49:1-15.

3. Harnoss JM, Strowitzki MJ, Radhakrishnan P, et al.: Therapeutic inhibition of prolyl hydroxylase domain-containing enzymes in surgery: putative applications and challenges. Hypoxia (Auckl) 2015; 3: 1-14.

4. Beck J, Henschel C, Chou J, Lin A, Del Balzo U: Evaluation of the carcinogenic potential of roxadustat (FG-4592), a small molecule inhibitor of hypoxia-inducible factor prolyl hydroxylase in CD-1 mice and sprague dawley rats. Int J Toxicol 2017; 36: 427-439.

5. Sugahara M, Tanaka T, Nangaku M: Prolyl hydroxylase domain inhibitors as a novel therapeutic approach against anemia in chronic kidney disease. Kidney Int 2017: 92: 306-312.

PD Dr. phil. II Rok Humar

Universitätsspital Zürich

Departement Innere Medizin

Wagistrasse 12

8952 Zürich-Schlieren

rok.humar@usz.ch 